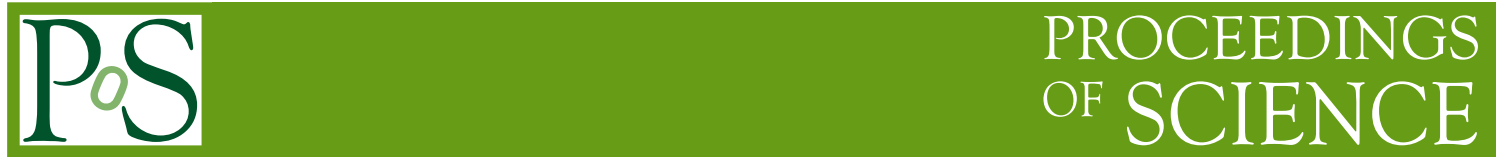

\title{
Cosmic-ray acceleration by compressive plasma fluctuations in supernova shells
}

\author{
Ming Zhang* \\ Florida Institute of Technology \\ E-mail: mzhang@fit.edu
}

\begin{abstract}
We suggest that the production of Galactic cosmic rays in supernova remnants undergoes two stages of acceleration mechanisms. Cosmic rays are first accelerated to certain cut-off energy that is significantly below the knee energy by the shock front. They then are further accelerated stochastically by compressive plasma fluctuations in the downstream region to the knee energy. If the compressive plasma fluctuations appear as an ensemble of shocklets, the stochastic acceleration process raises the cut-off energy without changing the shock power-law spectrum. In this way, the nonlinear shock acceleration effects become less severe, and it is not necessary to amplify upstream interstellar magnetic field to $\mathrm{mG}$ in order to make cosmic rays reach the knee energy.
\end{abstract}




\section{Introduction}

For a population of particles with a nearly isotropic distribution, the rate of particle momentum ( $p$ ) change averaged over all the directions is $d p / d t=\nabla \cdot \mathbf{V} p / 3$, where $\mathbf{V}$ is the velocity of background plasma embedded with magnetic fields. Since the divergence of plasma velocity $\nabla \cdot \mathbf{V}$ is a measure of plasma of compression or expansion, the behavior of particle energy gain or loss is directly tied to the compression of plasma media that they pass through. Particles gain energy whenever they go through regions of plasma compression with $\nabla \cdot \mathbf{V}<0$, and they are decelerated in plasma rarefaction regions where $\nabla \cdot \mathbf{V}>0$. We usually call this mechanism adiabatic heating or cooling because it is the same as that derived from the adiabatic process of the ideal gas in thermodynamics. The motion of plasma always contains some random fluctuations, so $\mathbf{V}=\mathbf{U}+\mathbf{u}$, where $\mathbf{U}$ is the average large-scale plasma flow velocity and $\mathbf{u}$ is the random small-scale component. In the limit of small fluctuations, the quasilinear approximation leads the random energy gain or loss to a diffusion process in the momentum space. The momentum diffusion coefficient can be calculated from the spectrum of longitudinal (in the same direction of the wavenumber $\mathbf{k}$ vector) plasma velocity fluctuation (Ptuskin 1988; Bykov and Toptygin 1993):

$$
D_{p p}=\frac{p^{2} 8 \pi \kappa}{9} \int_{0}^{\infty} d k \frac{k^{4} S(k)}{V_{w}^{2}+\kappa^{2} k^{2}},
$$

where we have assumed that the spectral density is isotropic, $S(k)$ is the velocity fluctuation spectral power density in $\mathbf{k}$ vector space, $\kappa$ is particle diffusion coefficient, and $V_{w}$ is the propagation speed of the fluctuation. In a plasma with small velocity fluctuations, the propagation speed of fluctuation $V_{w}$ is essential the phase speed of magnetosonic wave or sonic wave if the magnetic field is weak.

The stochastic particle acceleration by compressive plasma fluctuations is ubiquitous in space. Ptuskin (1998) applied the mechanism to cosmic rays propagating in the interstellar medium. He found that it is only marginally effective for the reacceleration of cosmic rays between $0.1-10 \mathrm{GeV}$.

In this paper, we apply this stochastic particle acceleration mechanism to cosmic rays in the downstream region of supernova remnant shock waves. Because of special conditions of in the downstream turbulent region, we argue that cosmic rays are accelerated to the knee energy of $3 \times 10^{15} \mathrm{eV}$ by the stochastic acceleration rather than by the shock wave in the front. A more extensive study on this subject using various turbulence spectra can be found in Zhang (2015).

\section{Estimate of acceleration time}

In order to estimate the time of particle acceleration, we need to know the spectrum of plasma velocity fluctuations. We assume that $S(k)$ is a power law of slope $-4+\alpha$ with $\alpha<1$ between $k_{0}=1 / L_{0}$ and $k_{1}=1 / L_{1}\left(L_{0} \gg L_{1}\right)$. A case of $\alpha=0$ corresponds to an ensemble of randomly oriented shock waves. Since the average velocity fluctuation is

$$
\left\langle u^{2}\right\rangle=4 \pi \int_{k_{0}}^{k_{1}} S(k) k^{2} d k
$$

After normalization to the total fluctuation, the spectrum $S(k)$ can be written as

$$
S(k)=\frac{(1-\alpha)\left\langle u^{2}\right\rangle}{4 \pi L_{0}^{1-\alpha}} k^{-4+\alpha} \text { for } k_{0}<k<k_{1}
$$




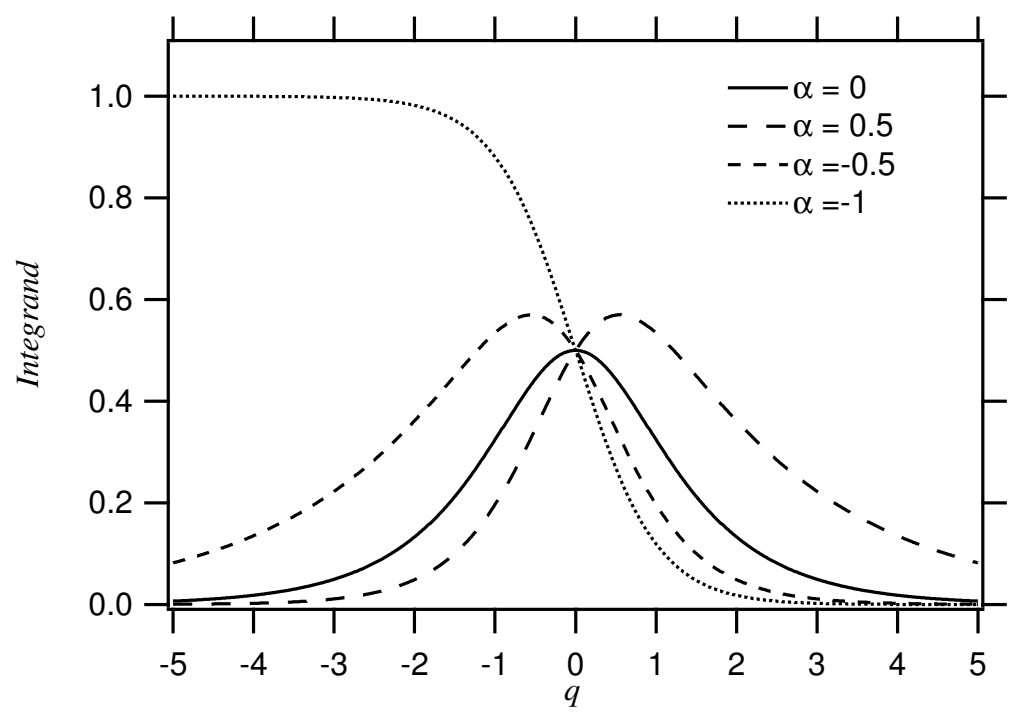

Figure 1: Integrad in $\mathrm{Eq}(2.3)$ as a function of varible $q=\ln \left(k \kappa / V_{w}\right)$

The time scale of particle acceleration $\left(\tau_{a c c}\right)$ can be estimated using $\tau_{a c c}=p^{2} / D_{p p}$ First, we look at what wavelength range of plasma velocity fluctuation contributes to the particle acceleration. Change variable $k=V_{w} \exp (q) / \kappa$, so that

$$
\tau_{a c c}^{-1}=\frac{2\left\langle u^{2}\right\rangle}{9 V_{w}^{1-\alpha} \kappa^{\alpha} L_{0}^{1-\alpha}} \int_{q_{0}}^{q_{1}} d q \frac{e^{(1+\alpha) q}}{1+e^{2 q}}
$$

Figure 1 shows some typical variations of the integrand in $\mathrm{Eq}(2.3)$ as a function of variable $q$. The integrand peaks at $q_{\max }=\ln [(1+\alpha) /(1-\alpha)] / 2$ for all $\alpha>-1$, suggesting the velocity fluctuation contribute to the particle acceleration the most at wavenumber $k_{\max }=\sqrt{(1+\alpha) /(1-\alpha)} V_{w} / \kappa$. For an $\alpha=0$, the maximum value of $1 / 2$ is located at at $q_{\max }=0$ or $k_{\max }=V_{w} / \kappa$, and its half maximum half width is $\Delta q=\ln (2+\sqrt{3})=1.3$ meaning the fluctuations between $k_{\text {low }}=(2-\sqrt{3}) V_{w} / \kappa$ and $k_{u p}=(2+\sqrt{3}) V_{w} / \kappa$ have a significant contribution to the acceleration of particles with a spatial diffusion coefficient $\kappa$. This analysis essentially tells us that the stochastic acceleration by compressive plasma fluctuations occurs in a broad "resonance" manner. If the power-law spectrum covers most of the resonance region with $q_{0} \ll q_{\max }-\Delta q$ and $q_{1} \gg q_{\max }+\Delta q$, then the integration is essentially the same as that one covering the entire range from $-\infty$ to $\infty$, yielding a value of $\pi / 2$. When $\alpha \neq 0$ but only slightly deviates from 0 , the integration in $\mathrm{Eq}(2.3)$ is close to $\pi / 2$, which leads to an acceleration time

$$
\tau_{a c c}^{-1} \approx \frac{\pi\left\langle u^{2}\right\rangle}{9 \kappa}\left(\frac{\kappa}{V_{w} L_{0}}\right)^{1-\alpha}
$$

In a case of $\alpha=0$, the acceleration is independent of particle diffusion coefficient $\kappa$, meaning that all particles are accelerated at the same rate as long as the $V_{w} / \kappa$ falls in between $q_{0}$ and $q_{1}$.

Now let us estimate for the time scale of particle acceleration. As an exercise, we first apply to cosmic rays in the ambient interstellar medium filled with an ensemble of shock waves due to relative motions of interstellar clouds. Typical diffusion coefficient of cosmic rays is $\kappa=10^{28}$ $\mathrm{cm}^{2} / \mathrm{s}$. In a warm medium of a $8 \times 10^{3} \mathrm{~K}$ temperature, the sound speed $V_{w}$ is $10.5 \mathrm{~km} / \mathrm{s}$, but in a hot 
medium of $10^{6} \mathrm{~K}, V_{w}=117 \mathrm{~km} / \mathrm{s}$. Cosmic rays are most effectively accelerated by compressive fluctuations of wavelengths in the range of 277 to $3092 \mathrm{pc}$. Take a $u=20 \mathrm{~km} / \mathrm{s}$ for the average relative motion between interstellar clouds, we can obtain an acceleration time $\tau_{a c c}$ ranging from $2.0 \times 10^{8}$ to $2.5 \times 10^{9}$ years. Compared to the confinement time of $1 \times 10^{8}$ for cosmic rays at $\sim 1 \times$ $10^{9} \mathrm{eV}$ as inferred from the measurements of radioactive isotope composition, these numbers for the acceleration time indicate that stochastic compressive acceleration is just marginally important to the reacceleration of cosmic rays in the interstellar medium.

The situation in the heated and magnetically amplified medium downstream of supernova remnant shocks are very different. For a supercritical supernova shock propagating at $U_{1}=10^{4}$ $\mathrm{km} / \mathrm{s}$ into the interstellar medium, the sound speed in the downstream region $V_{w}=\sqrt{5} U_{1} / 4=$ $5.6 \times 10^{3} \mathrm{~km} / \mathrm{s}$. The particle diffusion coefficient is most likely to be the Bohm diffusion limit because of the high level of plasma turbulence in the downstream media, i.e., $\kappa=p c /\left(3 Z e B_{2}\right)$, where $Z e$ is the charge of the particle and $B_{2}$ is the strength of magnetic field in the downstream media. The "resonant" wavelength of compressive plasma fluctuations occurs at

$$
L_{\max } \approx \frac{p c}{3 Z e B_{2} V_{w}}
$$

Across the supernova shock, the magnetic field is greatly amplified from its interstellar values due to plasma instability at the supercritical shock in addition to shock plasma compression. Observations (e.g., Vink \& Laming, 2003) suggest that the magnetic field behind the shock could be strong in the order of mG. Taking a $B_{2}=1 \mathrm{mG}$, we have $L_{\max }=1 \times 10^{-9} \mathrm{pc}$ for $1 \times 10^{9} \mathrm{eV}$ protons and $L_{\max }=3 \times 10^{-3}$ for $3 \times 10^{15} \mathrm{eV}$ protons at the knee energy of Galactic cosmic ray spectrum. These scales are much less than the size of the supernova remnant. It is possible that turbulence in the supernova shells can generate enough compressive plasma fluctuations of these scales. If the spectrum of compressive turbulence has a $k^{-4}$ slope or $\alpha=0$, the acceleration time can calculated through Eq (2.4), yielding an energy-independent acceleration time of $\tau_{a c c}=291$ years for all cos-

mic rays. In the calculation we have assumed that the amplitude of velocity fluctuations is equal to 0.3 times of bulk plasma flow behind the shock, or $u=2250 \mathrm{~km} / \mathrm{s}$ and $L_{0}=10 L_{\max }=3 \times 10^{-2} \mathrm{pc}$. Such a short time, which is less than the time for the supernova remnant to reach the Sedov-Taylor phase, suggests that stochastic acceleration by compressive fluctuations in the downstream region of supernova shocks can accelerate Galactic cosmic rays to the knee energy.

\section{Calculation of the energy spectrum of Galactic cosmic rays}

Most Galactic cosmic rays are accelerated from interstellar medium material by the forward supernova shock. After a certain amount of time, as power-law spectrum is established up to a cut-off energy $\left(E_{c}\right)$ or momentum $\left(p_{c}=E_{c} / c\right)$, which can be determined from the available time (Lagage \& Cesarsky, 1983)

$$
t=\int_{0}^{p_{c}} \frac{3}{U_{2}-U_{1}}\left(\frac{\kappa_{1}}{U_{s h}-U_{1}}+\frac{\kappa_{2}}{U_{s h}-U_{2}}\right) \frac{d p}{p}
$$

where $U_{s h}$ is the shock speed, $U_{1}(=0), U_{2}$ are plasma speed, and $\kappa_{1}, \kappa_{2}$ are the particle diffusion coefficient in the upstream (subscript 1) and downstream (subscript 2) media. Since $\kappa_{2} \ll \kappa_{1}$, the 
cut-off momentum is mainly determined by the upstream condition. Using the Bohm limit and typical magnetic field strength in the upstream interstellar magnetic field, the cut-off energy $E_{c}$ is estimated to be well below the knee energy in the cosmic ray spectrum. Adiabatic cooling in the expansion of supernova remnant further reduces the cut-off energy. A remedy was proposed to amplify the upstream interstellar magnetic field dramatically to a level greater than $\mathrm{mG}$ through a nonlinear mechanism of non-resonant instability induced by accelerated particles from the shock (Bell, 2004; 2005). There are many follow-up studies on this subject (e.g., Caprioli and Spitkovsky, 2014), but how the instability evolves nonlinearly into that high field and on a large scale (approximately the gyroradius of cosmic rays at the knee energy) in the upstream interstellar medium has yet been clearly demonstrated or understood. Besides, if the non-linearity is that strong to amplify upstream interstellar magnetic field to $\mathrm{mG}$, calculations show that the cosmic ray spectrum is no longer a power law (Bykov et al, 2014), which is not consistent with observations.

We suggest that stochastic acceleration my compressive plasma fluctuations in the downstream region is important to the production of comic rays. Cosmic rays gone through shock acceleration are further accelerated as it convects downstream. Neglecting the spatial diffusion $\kappa_{2}$, the particle distribution is governed by

$$
\frac{D f}{D t}=\frac{\partial f}{\partial t}+U \frac{\partial f}{\partial r}=\frac{\partial}{p^{2} \partial p}\left(D_{p p} p^{2} \frac{\partial f}{\partial p}\right)+\frac{1}{3} \frac{\partial}{r^{2} \partial r}\left(r^{2} U\right) p \frac{\partial f}{\partial p}
$$

Cosmic rays are injected at the shock at time $t_{0}$ with a distribution function $f_{0}\left(t_{0}, p\right)=A p^{-\gamma} H\left(p_{c}\left(t_{0}\right)-\right.$ $p$ ) with a shock spectral slope $\gamma=3 R /(R-1)$ where $R$ is the shock compression ratio (Drury, 1983). The normalization constant $A$ represents the particle intensity level.

Cosmic rays suffer an adiabatic cooling inside the supernova shell at a rate of $C=\frac{1}{3} \frac{\partial}{r^{2} \partial r}\left(r^{2} U\right)$. Using a linear dependence of plasma speed inside the shell, $U=U_{2} r / R_{s h}$, where the downstream plasma speed $U_{2}=U_{s h}(1-1 / R)$ and the radius of shock $R_{s h}=U_{s h} t$ for the free expansion phase, we have $\left.C=U_{2} / R_{s h}=(1-1 / R) / t=3 \gamma^{-1} / t\right)$, which decreases with the age of the supernova remnant.

The turbulence in the supernova shell should be strong after the plasma goes through the shock transition. Although most of the turbulence is in incompressible modes, a fraction of turbulence could be in the compressible model Incompressible turbulence could also be converted into compressible one during the evolution. As time involves and the supernova remnant cools down, the turbulence becomes more and more supersonic. The collision of flows leads to the formation of sheets of shocklets with a filamentary density distribution. We assume that the compression turbulence consists of an ensemble of shocklets. Then the spectrum of the velocity fluctuation is proportional to $k^{-4+\alpha}$ with an $\alpha=0$. In this case, Eq (refeq1) leads to a $D_{p p}=D_{0} p^{2}$, where $D_{0}$ is a constant equal to the rate of stochastic acceleration $\tau_{a c c}^{-1}$ in $\operatorname{Eq}(2.4)$. For mathematical simplicity, let us set up a time-dependent $D_{0}=D_{00} C=D_{00} 3 \gamma^{-1} / t$, which is also inversely proportional to the age $t$. Eq (3.2) in the comoving reference frame becomes:

$$
\frac{\gamma t}{3} \frac{D f}{D t}=\frac{\partial}{p^{2} \partial p}\left(D_{00} p^{4} \frac{\partial f}{\partial p}\right)+p \frac{\partial f}{\partial p}
$$

This diffusion equation can be solved analytically, yielding:

$$
f=A e^{3\left[(\gamma-3) D_{00}-1\right] \ln \left(t / t_{0}\right)} p^{-\gamma} \operatorname{erfc}\left(x_{c}\right)
$$


where $\operatorname{erfc}\left(x_{c}\right)$ is the complementary error function of variable

$$
x_{c}=\frac{\ln \left(p / p_{c}\right)-3 \gamma^{-1}\left[(2 \gamma-3) D_{00}-1\right] \ln \left(t / t_{0}\right)}{\sqrt{12 D_{00} \gamma^{-1} \ln \left(t / t_{0}\right)}} .
$$

Several important features of the solution to particle distribution function in $\mathrm{Eq}(3.4)$ should be pointed out. Below $x_{c}=0$ or below a certain energy all the spectra have a power-law spectrum that has the same spectral slope as the original shock spectrum. It means that stochastic acceleration in the downstream region does not alter the shock spectrum, independent of the stochastic acceleration rate or adiabatic cooling rate. The power-law spectrum cuts off at $x_{c}=0$, where the intensity falls below $1 / 2$ the expected power-law level, the new cut-off energy $E_{c}^{\prime}$ after stochastic acceleration and also adiabatic cooling is:

$$
E_{c}^{\prime}=\left(\frac{t}{t_{0}}\right)^{3 \gamma^{-1}\left[(2 \gamma-3) D_{00}-1\right]} E_{c}
$$

The intensity level of the power-law spectra is now different with a new normalization

$$
A^{\prime}=\left(\frac{t}{t_{0}}\right)^{3\left[(\gamma-3) D_{00}-1\right]} A .
$$

$D_{00}=D_{0} / C$ is the ratio of stochastic acceleration rate to adiabatic cooling rate. It determines the importance of the stochastic acceleration process during the evolution of the supernova remnant. In fact, as we estimated earlier, $D_{0}=\tau_{a c c}^{-1}=1 / 291$ year $^{-1}$ could be time-independent at least for the free expansion phase. The importance of the stochastic acceleration could evolve with the supernova remnant age. Early on, when $D_{00}<(2 \gamma-3)^{-1}=0.2$ or $t<3 \tau_{a c c} /[(2 \gamma-3) \gamma]=44$ years for a $\gamma=4$, both $E_{c}^{\prime}$ and $A^{\prime}$ decrease with time $t$. In this phase the adiabatic cooling dominates. If $(2 \gamma-3)^{-1}<D_{00}<(\gamma-3)^{-1}$ or 44 years $=3 \tau_{a c c} /[(2 \gamma-3) \gamma]<3 \tau_{a c c} /[(\gamma-3) \gamma]=218$ years, the cut-off energy $E_{c}^{\prime}$ increase with the age $t$ although the intensity $A^{\prime}$ decreases with the age $t$. During this stage stochastic acceleration play a weak role, but even so the cut-off energy still increases. After $t=3 \tau_{a c c} /[(\gamma-3) \gamma]=218$ years, $D_{00}>(\gamma-3)^{-1}$ or $D_{0}>C$, meaning the rate of stochastic acceleration exceeds the rate of adiabatic cooling. Both the particle intensity $A^{\prime}$ and cut-off energy $E_{c}^{\prime}$ increase with the age. During this phase the total pressure or energy density of the accelerated particles increase rapidly with $t$. Since cosmic rays already have taken up a significant fraction of total energy of supernova remnant, rapid energy conversion from the plasma turbulence to cosmic rays cannot sustain without modification of turbulence power by back-reaction of the further accelerated cosmic rays. So the system must return to no further growth of cosmic-ray intensity $A^{\prime}$, which requires $D_{00} \leq(\gamma-3)^{-1}$, or $D_{0} \leq C$. This stage is called critical stochastic acceleration or pressure balance stage. A initially strong enough compressive turbulence must lowers its power to ensure its effect on particle acceleration does not result in a rate faster than the rate of adiabatic cooling. This phase will last as long as the supernova shock is strong enough to generate enough compressive turbulence. The growth of cut-off energy will mainly occur in this stage of critical stochastic acceleration or pressure balance. Substituting $D_{00}=(\gamma-3)^{-1}$ into $\mathrm{Eq}(3.6)$, we have:

$$
E_{c}^{\prime}=\left(\frac{t}{t_{0}}\right)^{3 \gamma^{-1}\left[(2 \gamma-3)(\gamma-3)^{-1}-1\right]} E_{c}
$$


For a $\gamma=4, E_{c}^{\prime}=13 E_{c}$ after $t=2 t_{0}$. It can easily bring up the cut-off energy of the cosmic ray spectrum to the knee energy where the particles "resonate" with compressive plasma fluctuations of the longest wavelength $L_{0}$. Beyond the knee energy, there is little "resonant" power in the fluctuations to yield a large enough $D_{0}$. Because the acceleration only adds particles to the highest energy end of the spectrum where the particle density is low due to the steeply decreasing spectrum, the growth of the cut-off energy does not contribute much to the growth of total pressure, thus leaving the total energy of the system in check.

\section{Conclusion}

We suggest that stochastic acceleration by compressive plasma fluctuations is important to the production of Galactic cosmic rays in supernova remnants. Particles accelerated up to a certain cut-off energy below the knee energy by supernova shocks are further accelerated by the plasma fluctuations in the downstream region. If the fluctuations exist in the form of the randomly oriented shocklets ensemble, the mechanism can accelerate cosmic rays to the knee energy without changing the shock power-law spectrum, and in the meantime, it tends to raise cosmic ray intensity by canceling the effect of adiabatic cooling. In this way, the burden of accelerating Galactic cosmic rays on supernova shocks is reduced. Severe nonlinear shock acceleration effects are avoided and strong magnetic field amplification in the upstream interstellar medium is no longer necessary.

\section{References}

[1] A. R. Bell, Turbulent amplification of magnetic field and diffusive shock acceleration of cosmic rays, MNRAS, 353, (550), 2004.

[2] A.R. Bell, The interaction of cosmic rays and magnetized plasma, MNRAS, 358, (181), 2005

[3] A.M. Bykov, and I.N. Toptygin, REVIEWS OF TOPICAL PROBLEMS: Particle kinetics in highly turbulent plasmas (renormalization and self-consistent field methods), PhyU, 36, (1020), 1993.

[4] A. M. Bykov, D.C. Ellison, S. M. Osipov, A. E. Vladimirov, 2014, Magnetic Field Amplification in Nonlinear Diffusive Shock Acceleration Including Resonant and Non-resonant Cosmic-Ray Driven Instabilities, Astrophys. J., 789, (137), 2014.

[5] D. Caprioli, and A. Spitkovsky, Simulations of Ion Acceleration at Non-relativistic Shocks. II. Magnetic Field Amplification, Astrophys. J., 794, (46), 2014.

[6] L.Oc. Drury, An introduction to the theory of diffusive shock acceleration of energetic particles in tenuous plasmas, Rep. on Prog. Phys, 46, (973), 1983.

[7] P.O. Lagage, and C.J. Cesarsky, The maximum energy of cosmic rays accelerated by supernova shocks. Astron. \& Astrophys., 125, (249), 1983.

[8] V.S. Ptuskin, Cosmic-Ray Acceleration by Long-Wave Turbulence, SvAL, 14, (255), 1988

[9] J. Vink, \& J. M. Laming, On the Magnetic Fields and Particle Acceleration in Cassiopeia A, Astrophys. J., 548, (758), 2003.

[10] M. Zhang, Stochastic Acceleration of Galactic Cosmic Rays by Compressible Plasma Fluctuations in Supernova Shells, Astrophys. J., 812, (148), 2015. 